\title{
Intelligence heuristics to solve a Balanced Routing Problem in Supply Chain
}

\author{
N. Kannan \\ Professor \& Principal \\ Department of Computer Science \\ and Engineering \\ Jayaram college of Engineering \\ and Technology \\ Thuraiyur, Trichy, Tamilnadu, \\ India
}

\author{
S. Jayanthi \\ Associate Professor \\ Department of Computer Science \\ and Engineering \\ Saveetha school of Engineering \\ Chennai, Tamilnadu, India
}

\author{
R. Dhanalakshmi \\ Associate Professor \\ Department of Computer Science \\ and Engineering \\ National Institute of Technology \\ Nagaland, Dimapur- 797103, \\ India
}

\begin{abstract}
In today's business system, supply chain competitiveness greatly depends on its capability to handle the challenges of cost reduction, service level improvement and quality enhancement. In this competitive market, customer service level is the most significant factor for the success of the firm. Supply chain network needs to be efficient enough to handle the changing demand patterns. Balanced routing of goods among supply chain entities will improve the asset utilization and customer service level in a supply chain system. Over or under utilization of the supply chain entity will impact the customer service. We develop a decision support system based on three-stage heuristics to solve this balanced routing problem. A case study is illustrated and the DSS is validated for this case study.
\end{abstract}

\section{Keywords}

Balanced Routing Problem; Supply Chain System; Decision Support System

\section{INTRODUCTION}

The global and aggressive business environment has recognized the consequence of a quick and efficient service towards the customers in the past few decades. There is a need for uninterrupted flow of goods and materials between the supply chain entities (Chan and Kumar, 2007). The service level and performance of the supply chain network can be easily improved by balanced routing. Unbalanced routing can lead to the under or over utilization of assets and facilities and can further deteriorate the customer service. The logistics manager needs a decision support system (DSS) for operational decision-making of balanced routing. The main purpose of this paper is to develop a decision support system based on a heuristic for a balanced routing problem to run the business effectively in a dynamic system of supply chain. This problem is referred as balanced multiple traveling salesman problem (BMT), an extended version of multiple traveling salesman problem (mTSP). The heuristic is devised based on the idea proposed by Ganesh and Narendran (2007). The paper is organized as follows: Section 2 details the literature review. The problem is defined in Section 3. DSS based on heuristic is explained in Section 4. Results and Interpretations are explained in Section 5. Section 6 concludes the paper with the future scope of research.

\section{LITERATURE SURVEY}

Compared to the TSP, $m$ TSP relates to accommodate models with real life situations, since it is capable of handling more than one salesman. These situations arise mostly in various routing and scheduling problems. Some reported applications are Print press scheduling (Gorenstein 1970), Pre-print advertisement scheduling (Carter \& Ragsdale 2006), Workforce Bank crew scheduling (Svestka \& Huckfeldt 1973) Technical crew scheduling (Lenstra \& Rinnooy Kan 1975), Interview scheduling (Gilbert \& Hofstra 1992), Workload balancing (Okonjo-Adigwe 1988), Security service scheduling (Calvo \& Cordone 2003), Transportation School bus routing (Ledesma J. R, et al, 2012), Local truckload pickup and delivery (Wang \& Regan, 2002), Planning of autonomous mobile robots (Brumitt et al. 2001), Designing satellite surveying systems (Saleh \& Chelouah 2004). Therefore, finding a good methodology for the $m \mathrm{TSP}$ is important and induces to improve the solution of any other complex routing problems. However, the $m$ TSP is $N P$-complete as it includes the TSP as a special case. The $m T S P$ is more difficult than the TSP because it requires determining which cites to assign to each salesperson, as well as the optimal ordering of the cities within each salesperson's tour. Svestka \& Huckfeldt (1973) suggested transforming the $m$ TSP with $m$ salesmen and $n$ cities into a TSP with $n+m-1$ cities by the introduction of $m-1$ artificial depots $(n+1, \ldots, n+m-1)$. However, the resulting TSP has highly degenerated, when an $m \mathrm{TSP}$ is transformed into a single TSP since the resulting problem is more arduous to solve than an ordinary TSP with the same number of cities. A number of different methods have been proposed for different variants of $m$ TSP for obtaining either optimal or near optimal solutions for the TSP.

The single depot or multiple depot multiple-vehicle node routing problem (vehicle routing problem - VRP) prescribes a set of delivery routes for vehicles housed at a central depot, which services all the nodes and minimizes total distance traveled. The formulations of these problems were stated by Bodin et al. (1983). This problem can be extended by adding side-constraints such as time windows. There is a lot of interest among the researchers regarding freight transportation and empty container distribution. The planning horizon, the tactical planning, the effective forecasting, all came under one roof when freight transportation and empty container distribution are considered. Few procedures and important landmarks in the literature of transportation problem, especially freight transportation and vehicle routing problems are discussed here:

- Cluster-first route-second procedures, which cluster demand nodes and/or arcs first, then design, economical routes over each cluster as a second step. Example of this idea was given by Gillett \& Miller (1974). 
- $\quad$ Route - first cluster - second procedures, which work in a reverse sequence in comparison with cluster - first route - second. Golden et al. (1984) provided an algorithm that typifies this approach for a heterogeneous fleet size VRP.

- Savings or insertion procedures, which build a solution in such a way that at each step of the procedure, a current configuration that is possibly infeasible. The alternative configuration is one that yields the largest saving in terms of some criterion function, such as total cost, or that inserts least expensively a demand entity in the current configuration into the existing route or routes. Examples of these procedures can be found in Clarke \& Wright (1964) or in Solomon (1987).

- Improvement or exchange procedures, such as the wellknown branch exchange heuristic which always maintain feasibility and strive towards optimality. Other improvement procedures were described by Potvin \& Rousseau (1995), including Or-opt exchange method in which, first, second, third, consecutive nodes in a route will be removed and inserted at another location within the same or another route; k-interchange heuristic in which $\mathrm{k}$ links in the current routes exchange of $\mathrm{k}$ new links; and 2-opt procedure which exchanges only two edges taken from two different routes.
- Mathematical programming approaches, which include algorithms that are directly based on a mathematical programming formulation of the underlying routing problem. An example of this procedure was given by Fisher \& Jaikumar (1981). Christofides et al. (1981) discussed Lagrangean relaxation procedures for the routing of vehicles.

- Exact procedures for solving VRPs, which includes specialized branch and bound (B \& B), dynamic programming and cutting plane algorithms. Kolen et al. (1987) described a branch and bound (B \& B) method for the vehicle routing problem with time windows (VRPTW). Other applications of the above algorithms for VRPs were discussed in details by Christofides et al. (1981).

Chandran et al. (2006) discussed the detailed literature survey, application and types of mTSP. The balanced mTSP is a problem which was introduced by Chandran et al. (2006) and they developed a heuristic based on load balanced clustering and cluster length approximation algorithm. Parthiban et al. (2011) discussed the review of evolutionary algorithms for multi objective, multi product multimodal distribution problem with a possible new approach

Table 1: Literature Summary

\begin{tabular}{|c|c|c|}
\hline Variants & Methodology & Author and Year \\
\hline \multirow[t]{11}{*}{ MTSP } & Heuristic Approach & Gorenstein (1970) \\
\hline & Branch and Bound Algorithm & Svestka and Huckfeldt (1973) \\
\hline & Linear admissible transformations & Berenguer (1979) \\
\hline & Branch and bound algorithm & Radharamanan and Choi (1986) \\
\hline & Heuristic Approach & Okonjo-Adigwe (1988) \\
\hline & Multi stage Monte Carlo optimization & Conley (1990) \\
\hline & Tabu Search & Cordeau et al (1997) \\
\hline & Adaptive Neural Network & Torki (1997) \\
\hline & Competition based Neural Network & Somhom (1999) \\
\hline & Modified Genetic Algorithm & Tang et al, (2000) \\
\hline & Genetic Algorithm & Carter and Ragsdale (2006) \\
\hline mTSPMD & Integer Linear Programming & Kara and Tolga Bektas (2006) \\
\hline \multirow[t]{3}{*}{$m T S P T W$} & Decomposition Heuristic & Calvo and Cordone (2003) \\
\hline & Genetic Algorithm & Saleh and Chelouah (2004) \\
\hline & Graph Theory - Precedence Graphs & Mitrović-Minić and Krishnamurti (2006) \\
\hline$m T S P M P$ & Heuristic Approach & Gilbert and Hofstra (1992) \\
\hline
\end{tabular}




\section{PROBLEM DEFINITION}

The Traveling Salesman Problem (TSP) is arguably the most prominent problem in combinatorial optimization. The simple way in which the problem is defined in combination with its notorious difficulty has stimulated many efforts to find an efficient solution procedure. The TSP is a classic tour problem in which a hypothetical salesman must find the most efficient sequence of destinations in his territory, stopping only once at each, and ending up at the initial starting location. Due to the combinatorial complexity of the TSP, approximate or heuristic solution procedures are almost always employed in practice. The TSP arises in many different contexts and apparently unrelated problems are solved by formulating them as instances of the TSP, most applications originate in the real world problems like vehicle routing, clustering a data array, job-shop scheduling or computer wiring (Laporte and Osman, 1995 and Lawler et al. 1985). The multiple traveling salesman problem (mTSP) is a generalization of the TSP, with more than one salesman. Given a set of cities, with $m$ number of salesmen located at a single location called "depot city" and intermediate cities as remaining cities that are to be visited. The mTSP consists of finding tours for all $m$ salesmen, who all start and end at the depot-city. The constraints of movement are the same as found in the TSP in that each intermediate city is visited exactly once and the total cost of visiting all cities is minimized (Bektas 2006). We consider balanced multiple traveling salesman problem where the distance between the vehicles are balanced to improve the asset utilization and customer service level (Okonjo-Adigwe, 1988 and Chandran et al. 2006). All the salespersons has to start from a common depot and after traveling through a set of cities, they should return back to the starting depot. There are no capacity constraints and no cost constraints. But, all the cities must be visited by any one of the salesperson and each salesperson has to visit a particular city exactly once.

\section{DECISION SUPPORT SYSTEM BASED ON HEURISTIC- A CASE STUDY}

We develop a DSS based on three-stage heuristic to solve BMT. In the first stage, we cluster the nodes based on a clustering approach. In the second phase, we used shrink wrap algorithm proposed by Ganesh and Narendran (2007) to form the initial tour. In the third stage, we develop a simulated annealing (SA) approach to improve and balance the tour. The Flowchart representing the heuristic is given in Fig. 1.

DSS based on heuristic is applied for a school van routing case study. The details of the heuristic are explained with a case study. There are school 6 vans which needs to route through 60 places to pick-up the kids for school. The objective is to find the balanced routing with minimal cost.

\subsection{First Phase of Heuristic}

Clustering offers a major computational advantage, owing to the fact that the sum of factorials is much smaller than the factorial of the sum. For a routing problem with $\mathrm{N}$ cities, the search space is a function of $\mathrm{N}$ ! Thus the computing time, which is proportional to the search space, is also a function of $\mathrm{N}$ ! When $\mathrm{N}$ is a large number, the computing time is very high. While decomposing the problem, if $\mathrm{N}$ cities are divided into $\mathrm{P}$ clusters, the average number of cities in each cluster in $\mathrm{N} / \mathrm{P}$. Therefore, the search space for each cluster is a function of $(\mathrm{N} / \mathrm{P})$ ! The total search space for all clusters is: $\mathrm{P}(\mathrm{N} / \mathrm{P})$ ! When $\mathrm{N}$ is large, clustering saves time by several orders of magnitude, since

$$
P \times\left(\frac{N}{P}\right) ! \ll N !
$$

for large $\mathrm{N}$ and $\mathrm{P}$ (Anderberg, 1973). 


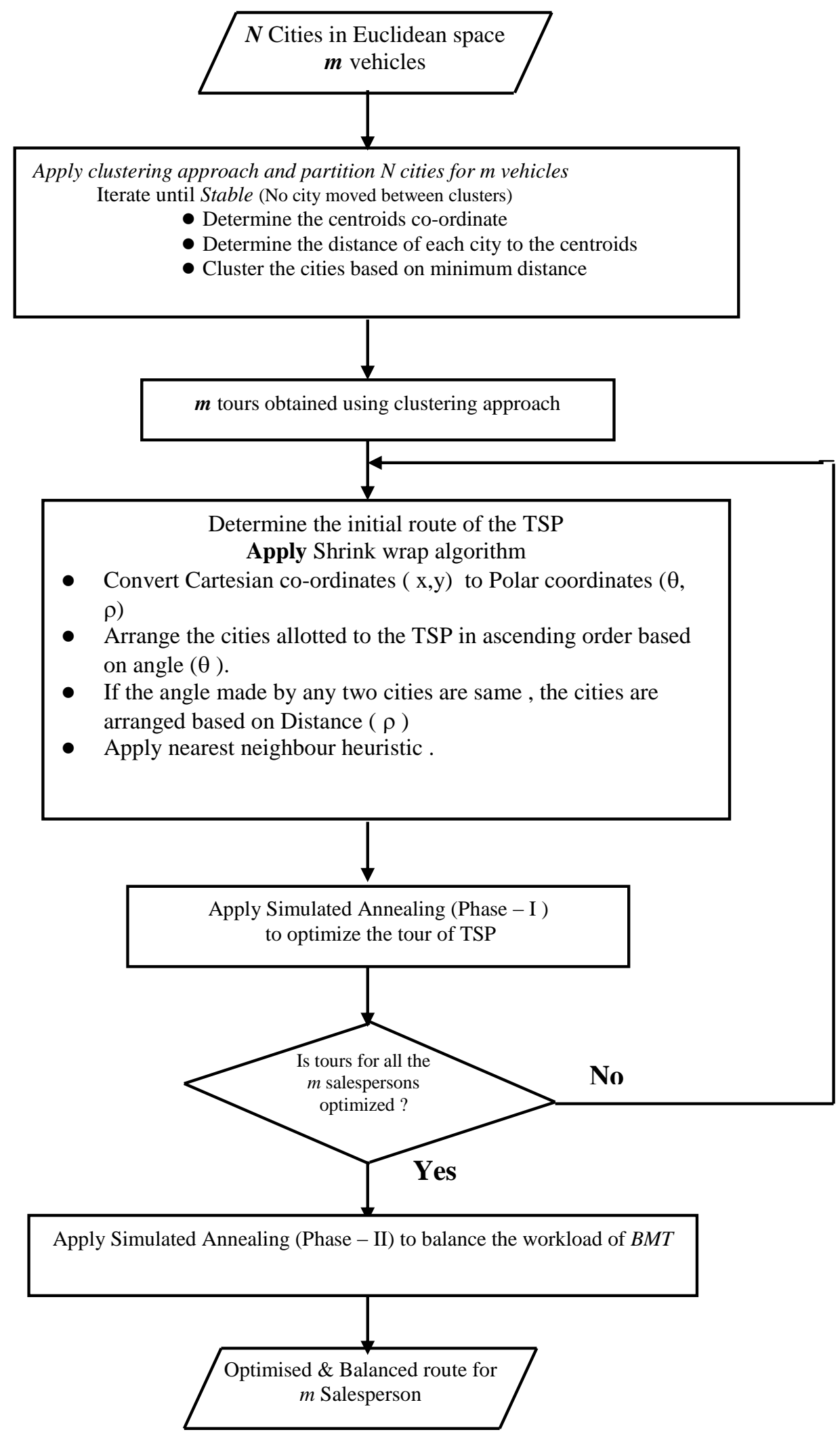

Figure1. DSS Based on Heuristic for BMT

\subsubsection{Clustering Approach}

The steps of the proposed clustering approach are as follows:

Step 1: Choose $\mathrm{K}$ initial cluster centers $\mathrm{Z}_{1}, \mathrm{Z}_{2}, \ldots . ., \mathrm{Z}_{\mathrm{k}}$ from the ' $\mathrm{n}$ ' points $\left\{\mathrm{X}_{1}, \mathrm{X}_{2}, \ldots \ldots, \mathrm{X}_{\mathrm{n}}\right\}$
Step 2 : Assign point $\mathrm{X}_{\mathrm{i}}, \mathrm{i}=1,2, \ldots, \mathrm{n}$ to cluster $\mathrm{C}_{\mathrm{j}}, \mathrm{j} \in\{1,2$, ...., $K$ \} iff $\left\|X_{i}-Z_{j}\right\|<\left\|X_{i}-Z_{p}\right\|, p=1,2, \ldots, K$, and $j \# p$

Ties are resolved arbitrarily 
Step 3: Compute new cluster centers $\mathrm{Z}_{1}^{*}, \mathrm{Z}_{2}{ }^{*}, \ldots, \mathrm{Z}_{\mathrm{k}}{ }^{*}$ as follows:

$$
Z_{i}^{*}=\frac{1}{n_{i}} \sum_{X_{j}=C_{i}} X_{j}, \quad i=1,2, \ldots, K,
$$

Where $n_{i}$ is the number of elements belonging to cluster $C_{i}$
Step 4: If $\mathrm{Z}_{\mathrm{i}}{ }^{*}=\mathrm{Z}_{\mathrm{i}}, \mathrm{i}=1,2, \ldots, \mathrm{K}$ then terminate. Otherwise continue from step 2.The input data for a case study is shown in

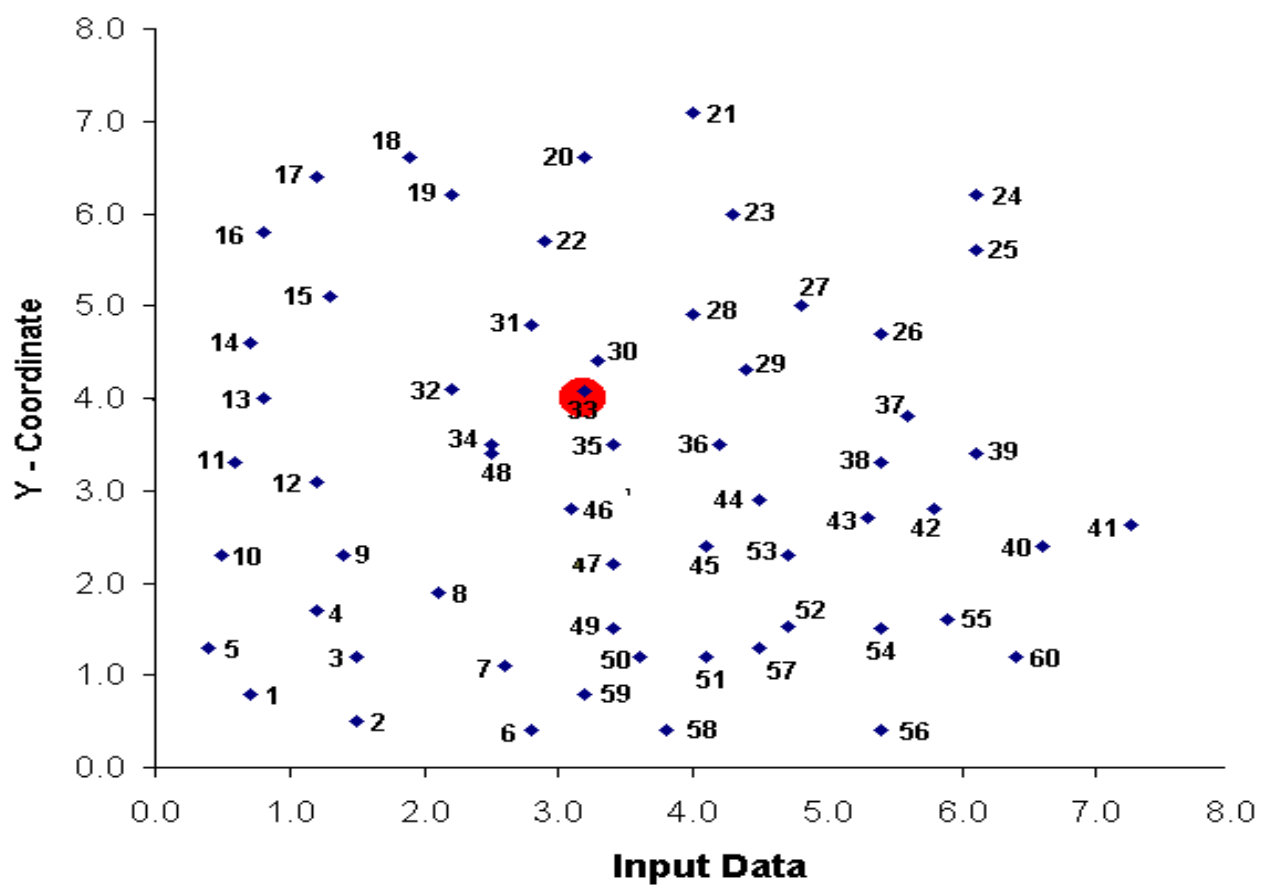

Figure2. Input Data for the Case Study

Although this algorithm must always converge, there is no known limit on the number of iterations required. The clustering approach is suitable for large sets of numeric objects despite the fact that it is computationally expensive. It is sensitive to the selection of the initial partition and may converge to a local minimum of the criterion function value if the initial partition is not properly chosen. The convergence is not guaranteed to yield a global optimum. The quality of the final solution depends largely on the initial set of clusters .Hence the following methodology is adopted to initialize the centroids. By arranging the cities based on distance calculated using the Euclidean distance formula,

$$
\sqrt{\left[\left(x_{i}-x_{2}\right)^{2}+\left(y_{1}-y_{2}\right)^{2}\right]}
$$

We find the cities 33, 30, 31 are very close to the depot and the cities 56,60, 41 are farthest from the depot considered. In the formulae, suffix $1 \& 2$ represents the cities between which distance is to be calculated. Here we have considered the depot itself as an initial centroid. The initial cluster centroids are given in the Table 2 .

Table 2: Initial Cluster Centroids

\begin{tabular}{|c|c|c|}
\hline Cluster No & X-coordinate & Y-coordinate \\
\hline 1 & 3.1 & 4.2 \\
\hline 2 & 3.3 & 4.5 \\
\hline 3 & 2.8 & 4.8 \\
\hline 4 & 5.4 & 0.4 \\
\hline 5 & 6.4 & 1.2 \\
\hline 6 & 7.7 & 2.6 \\
\hline
\end{tabular}

By applying the clustering approach, mTSP is transformed in to TSPs and the results are shown in Table 3 and Fig. 3 
Table3. Final Results of Clustering Approach

\begin{tabular}{|c|c|c|}
\hline Vans & Cities Allotted & No. of cities \\
\hline 1 & $1,3,4,5,8,9,10,11,12,13,34,46,48$ & 13 \\
\hline 2 & $21,23,24,25,26,27,28,29,30,35,36$ & 11 \\
\hline 3 & $14,15,16,17,18,19,20,22,31,32$ & 9 \\
\hline 4 & $2,6,7,47,49,50,51,58,59$ & 10 \\
\hline 5 & $43,44,45,52,53,54,55,56,57,60$ & 6 \\
\hline 6 & $37,38,39,40,41,42$ & \\
\hline
\end{tabular}

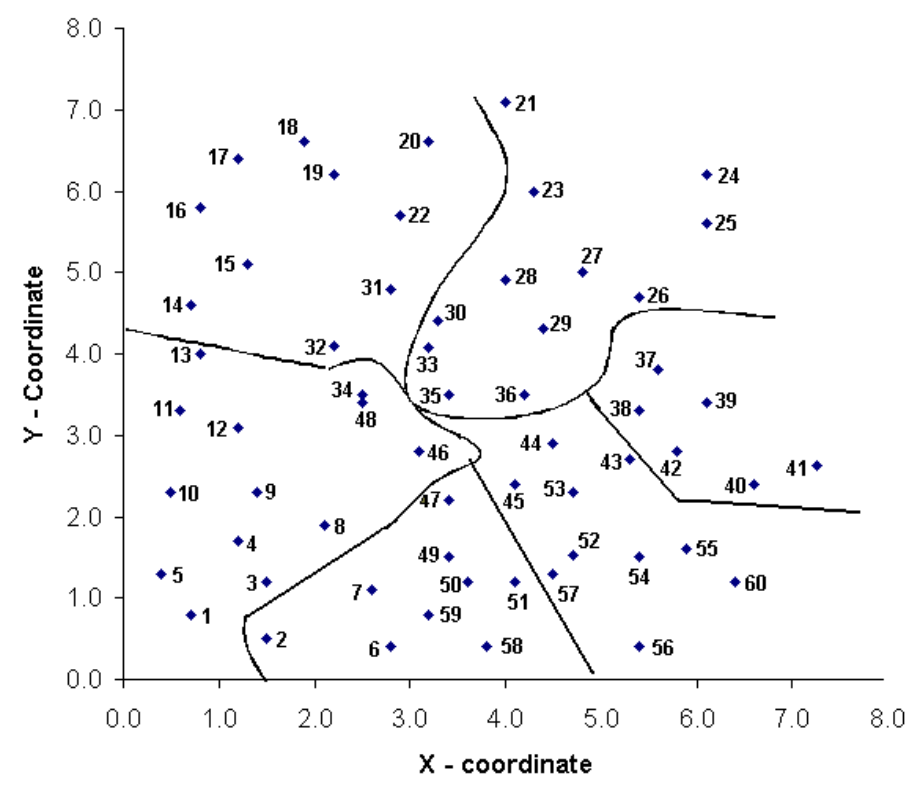

Figure 3. Output of Clustering Approach

\subsection{Second Phase of Heuristic- Shrink Wrap Algorithm}

In each cluster obtained, we orient the nodes along a path using the Shrink-Wrap Algorithm (Lawler et al. 1985, Ganesh and Narendran, 2007). The nodes are mapped on polar coordinates, sorted by angle $(\theta)$, then by distance $(\rho)$ and arranged in ascending order. This gives the path to be traversed within each cluster. Briefly, the steps are shown in the Fig. 4. So, cluster approach is used for generating clusters, and then shrink wrap algorithm is used to optimize the clusters and finally, the cluster is unwrapped using nearest neighbor heuristic. The results are given in Table 4 and the output is shown in Fig.5.

Table 4: Results of Shrink Wrap Algorithm Approach

\begin{tabular}{|c|c|c|}
\hline Vans & Route generated & $\begin{array}{c}\text { Distance to } \\
\text { travel }\end{array}$ \\
\hline 1 & $33-48-34-46-8-3-9-4-1-5-10-11-13-12-33$ & 13.4058 \\
\hline 2 & $33-30-28-21-23-24-25-27-26-29-36-35-33$ & 12.4839 \\
\hline 3 & $33-31-32-14-15-16-17-18-19-20-22-33$ & 10.3033 \\
\hline 4 & $33-47-49-50-51-58-59-6-2-7-33$ & 11.4210 \\
\hline 5 & $33-44-43-55-60-54-56-57-52-45-53-33$ & 12.5020 \\
\hline 6 & $33-38-37-39-41-40-42-33$ & 10.4913 \\
\hline
\end{tabular}




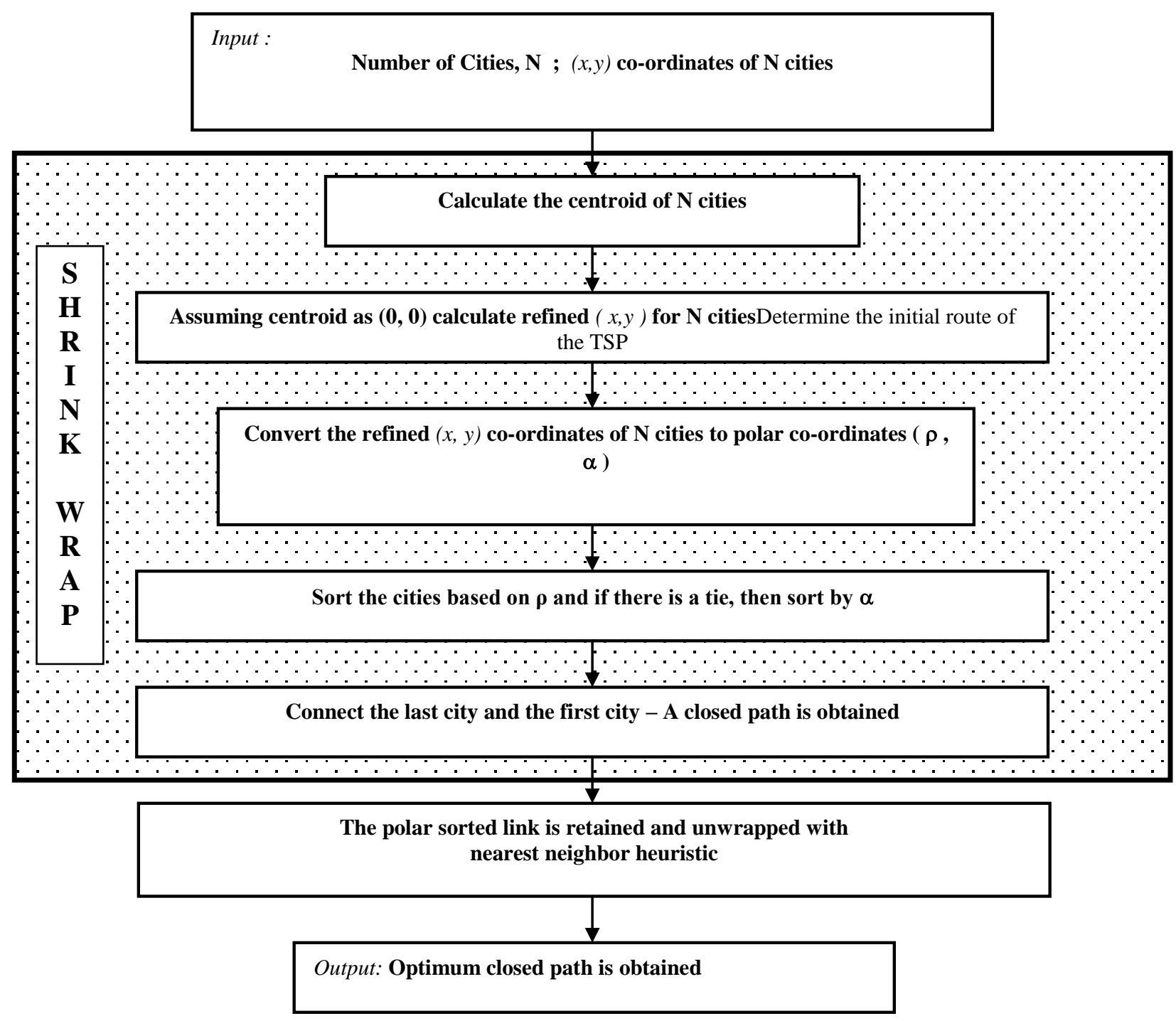

Figure. 4. Second Phase- Shrink wrap algorithm 


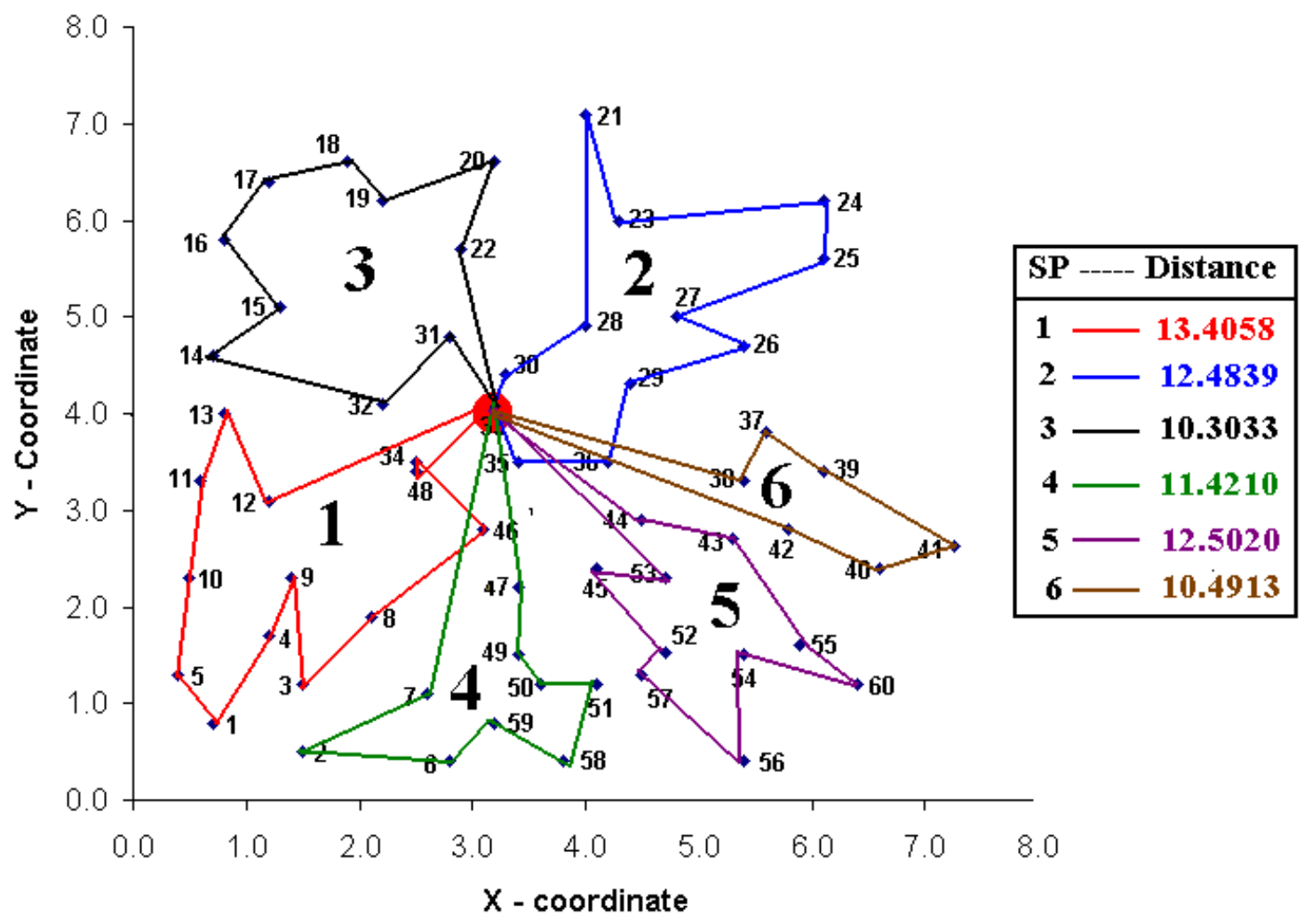

Figure5. Routing Results of Shrink-Wrap Algorithm

\subsection{Third Phase - Simulated Annealing for Improving and Balancing the Route}

The cities sequence obtained using the cluster analysis heuristic is considered as the chromosome for SA. The sequences are generated randomly and the sequences are improved with the concept of SA to arrive at the better solutions in a reasonable computation time. The classical concept of generating a sequence of solutions for a particular temperature $T$ is used in the algorithm. A new solution is generated as a slight perturbation of the current state by using a single insertion neighborhood creation scheme by creating 2(n-1) neighborhoods. If the difference in the total cost, $\delta$ between the current state and the slightly perturbed one is negative, then the process is continued from the new state. If $\delta$ $>=0$, then the perturbation of acceptance of the perturbed state is given by the Metropolis. For a given sequence $\sigma$, this neighborhood is defined as the set of sequences obtained by moving any product from its actual position in $\sigma^{1}$ to any other position. The neighbor solutions are generated randomly with the restriction on number of vehicles and capacity restriction of vehicles. The number of neighborhood depends upon the application and the common formula used is 2 (n-1) numbers of neighborhoods. From those neighborhoods the best one is selected according to the objective function.

The flow chart for SA is shown in the Fig.6 


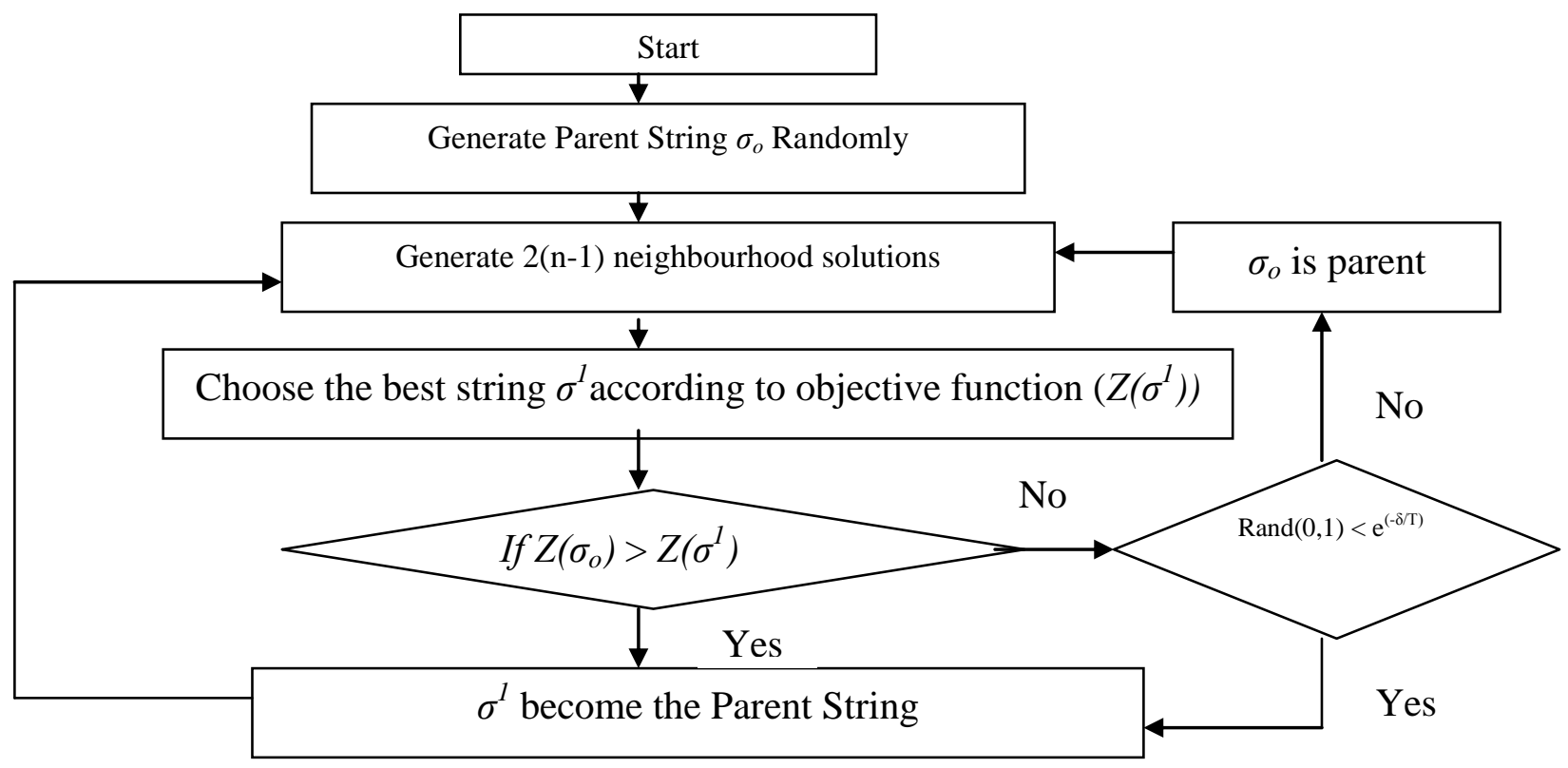

Figure6. Flow Chart for SA

The output obtained using the shrink wrap algorithm is improved using route improvement heuristic which applied the meta-heuristic - Simulated annealing. The algorithm for
SA is given below and the results (Table 5) obtained in this stage of the heuristic is given in Fig. 7

Table 5: Results of simulated annealing

\begin{tabular}{|c|c|c|}
\hline Vans & Route generated & $\begin{array}{c}\text { Distance to } \\
\text { travel }\end{array}$ \\
\hline 1 & $33-46-8-9-4-3-1-5-10-11-13-12-34-48-33$ & 12.3237 \\
\hline 2 & $33-30-28-23-21-24-25-26-27-29-36-35-33$ & 11.3353 \\
\hline 3 & $33-32-14-15-16-17-18-19-20-22-31-33$ & 9.6791 \\
\hline 4 & $33-47-49-50-51-58-59-6-2-7-33$ & 11.4210 \\
\hline 5 & $33-44-43-54-55-60-56-57-52-53-45-33$ & 11.3932 \\
\hline 6 & $33-37-39-41-40-42-38-33$ & 10.0836 \\
\hline
\end{tabular}




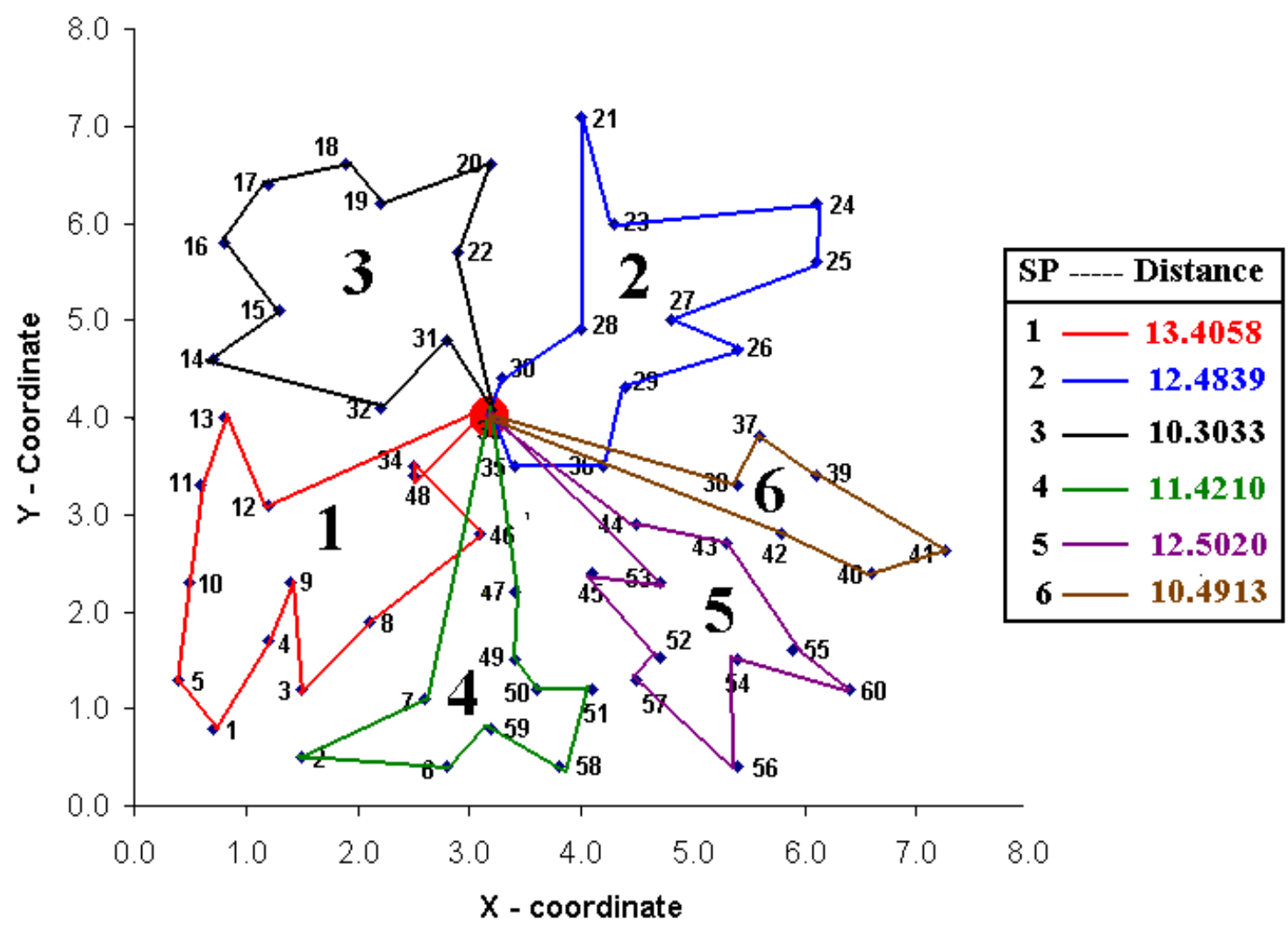

Figure7. Improved Solution by SA

\subsubsection{Balancing the Route using SA}

The optimized route obtained from the route improvement heuristic gives a path for the van to travel. But, it could be noticed that the distance traveled by the van were not equal. Since, this may decrease the morale of the employees, any employee oriented organization would like to balance their workload as far as possible. These differences in workload can be minimized by minimizing the standard deviation of the output. Standard deviation actually indicates the variability of a data around its mean. The higher the standard deviation, the greater the variability in data would be. Therefore, the objective of balancing heuristic is to minimize the standard deviation up-to lowest possible value. If the standard deviation is 0 , all the numbers are the same. But in balancing the distance, this may not be possible because, we may not get the exact distances for all the vans. Hence, we choose the criteria as minimizing the standard deviation up-to the lowest possible value (may be greater than zero) (Bedeian and Mossholder, 2000).
The standard deviation for this problem is given by

$$
s=\sqrt{\Sigma(X-\bar{X})^{2} /(n-1)}
$$

where,

$$
\begin{aligned}
& \mathrm{s}=\text { standard deviation } \\
& \mathrm{X}=\text { distance traveled by the van } \\
& \mathrm{n}=\text { number of vans. }
\end{aligned}
$$

Hence, by converging standard deviation to a minimum possible value, one can balance the workload. In this paper, we find the initial standard deviation as 1.065654 and converging the same using SA would give the minimum possible value as 0.470163 . The distance traveled by the van in this case is shown in Table 6 and Fig. 8

Table 6. Results of Balancing Rule of Third Phase SA

\begin{tabular}{|c|c|c|}
\hline $\begin{array}{c}\text { Sales } \\
\text { Person }\end{array}$ & \multicolumn{1}{|c|}{ Route generated } & $\begin{array}{c}\text { Distance to } \\
\text { travel }\end{array}$ \\
\hline 1 & $33-48-34-46-8-9-4-3-1-5-10-11-13-32-33$ & 11.7622 \\
\hline 2 & $33-30-31-23-21-24-25-26-27-29-36-35-33$ & 11.8929 \\
\hline 3 & $33-45-53-52-57-56-60-55-54-43-44-33$ & 11.3932 \\
\hline 4 & $33-47-49-50-51-58-59-6-2-7-33$ & 11.4210 \\
\hline 5 & $33-12-14-15-16-17-18-19-20-22-28-33$ & 11.8930 \\
\hline 6 & $33-37-39-41-40-42-38-33$ & 10.0836 \\
\hline
\end{tabular}




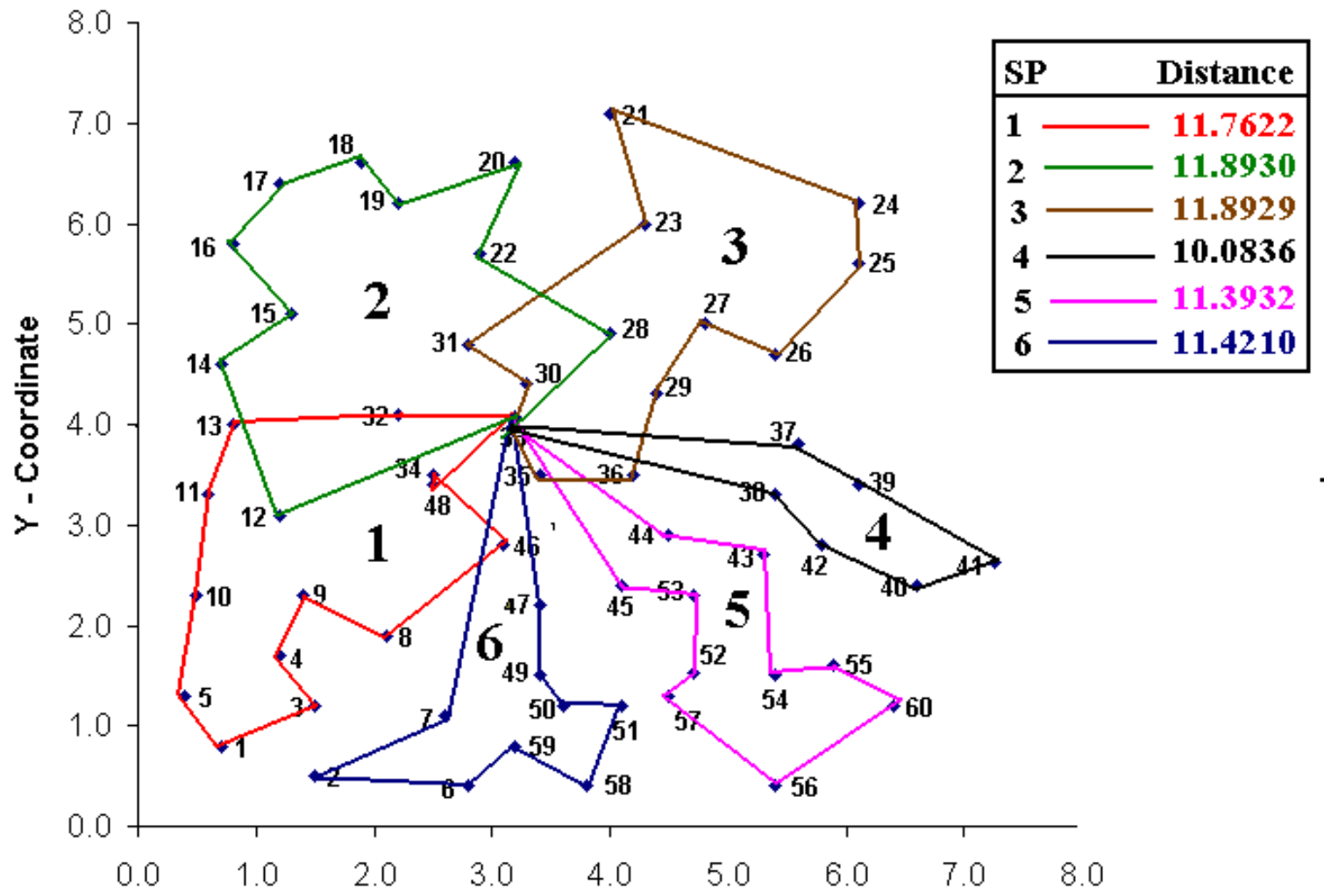

Figure 8. Balanced Route by SA

The sum of distances to be traveled by the six vans is 66.7322 (Optimum) and it is unbalanced. To balance the tour, the increase in the total tour length should be acceptable, but as far as possible this value should be minimum.

\section{RESULTS \& DISCUSSIONS}

In first phase, the initial seeds for clustering approach affects the output quality of the clusters formed and this has been solved by taking alternatively the nearest and farthest city locations as seeds. This forms a better approach than random selection. If second phase of the heuristic is skipped off, it may be noticed that achieving optimality for the van tour is difficult as the Simulated annealing (SA) is a very slow process and it takes more time to converge. Hence, Shrink wrap algorithm is applied to form the initial tour and it serves as the input for SA. Since the output of SA will not be of global minimum, the third phase of the heuristic helps us to rely upon SA as it takes less burden of solving a TSP. Hence, we can conclude that the SA can be applied to improve the optimality of the tour for BMT. The total distance to be traveled by the six vans reduced from 70.6073 to 66.2359 . After applying the balancing rule of the third phase of heuristic, it may be noticed that the total increase in distance is only $2.5 \%$ and the distances are almost balanced. The results comparisons are shown in Table 7.

Table 7. Comparison of Distances at Various Phases of Heuristic

\begin{tabular}{|l|l|l|l|}
\hline Vans & $\begin{array}{l}\text { Unoptimised } \\
\text { Distance }\end{array}$ & $\begin{array}{l}\text { Optimised } \\
\text { Distance }\end{array}$ & $\begin{array}{l}\text { Balanced } \\
\text { Distance }\end{array}$ \\
\hline $\mathbf{1}$ & 13.4058 & 12.3237 & 11.7622 \\
\hline $\mathbf{2}$ & 12.4839 & 11.3353 & 11.8929 \\
\hline $\mathbf{3}$ & 10.3033 & 9.6791 & 11.3932 \\
\hline $\mathbf{4}$ & 11.4210 & 11.4210 & 11.4210 \\
\hline $\mathbf{5}$ & 12.5020 & 11.3932 & 11.8930 \\
\hline $\mathbf{6}$ & 10.4913 & 10.0836 & 10.0836 \\
\hline TOTAL & $\mathbf{7 0 . 6 0 7 3}$ & $\mathbf{6 6 . 2 3 5 9}$ & $\mathbf{6 8 . 4 4 5 9}$ \\
\hline
\end{tabular}

\section{CONCLUSION}

This paper proposed a workable and simple method of solving a balanced multiple traveling salesperson problem (BMT) with the issue of balancing the workload in a reasonable time.
This heuristic balanced the workload with the increase in a tour length of only $2.5 \%$. Hence, this methodology finds direct or indirect method to solve practical applications such as reverse logistics, service logistics and many more. Most of the logistics issues will have pickup or only delivery or both 
pickup and delivery. This issue may be addressed by imposing many constraints in solving an mTSP. For deterministic models, this heuristic will be directly useful. But for stochastic models, the research is still needed to make it to be useful for Decision Support system (DSS).

\section{REFERENCES}

[1] Anderberg, M.R. (1973) 'Cluster analysis for applications', Academic press, New York.

[2] Bedeian, A.W. and Mossholder, K.W. (2000) 'On the Use of the Coefficient of Variation as a Measure of Diversity', Organizational Research Methods, Vol. 3, No. 3, pp. 285-297.

[3] Bektas, T. (2006) 'The multiple traveling salesman problem: an overview of formulations and solution procedures' Omega, In Press, Corrected Proof, Available online www.sciencedirect.com.

[4] Berenguer, X (1979) A characterization of linear admissible transformations for the $m$-travelling salesmen problem, European Journal of Operational Research. Volume 3, Issue 3, Pages 232-238.

[5] Bodin L, Golden B, Assad A, Ball M (1983). Routing and scheduling of vehicles and crews: The state of the art. Comput Oper Res 10: 63-211.

[6] Brumitt, B; Stentz, A; Hebert, M and The Cmu Ugv Group. (2001) Autonomous Driving with Concurrent Goals and Multiple Vehicles: Mission Planning and Architecture, Autonomous Robots 11, 103-115.

[7] Calvo, R.W. and Cordone, R. (2003) 'A heuristic approach to the overnight security service problem', Computers and Operations Research, Vol. 30, pp.12691287.

[8] Carter, A.E. and Ragsdale, C.T (2006). "A new approach to solving the multiple traveling salesperson problem using genetic algorithms", European journal of operational research, Vol.175, no.1, pp. 246-257.

[9] Chandran, N., Narendran, T.T. and Ganesh, K. (2006) 'A clustering approach to solve the multiple travelling salesmen problem', Int. J. Industrial and Systems Engineering, Vol. 1, No. 3, pp.372-387.

[10] Chan, F.T.S. and Kumar, N. (2007), 'Effective allocation of customers to distribution centres: A multiple ant colony optimization approach', Robotics and ComputerIntegrated Manufacturing, In Press, Corrected Proof, Available online 13 August 2007

[11] Christofides Nicos , A. Mingozzi and P. Toth (1981) State-space relaxation procedures for the computation of bounds to routing problems. DOI: 10.1002/net.3230110207 Volume 11, Issue 2, pages $145-164$.

[12] Clarke G and Wright J (1964). Scheduling of vehicles from central depot to number of delivery points. Operations Research, 12(4):568-581.

[13] Conley. W (1990) Multi-stage Monte Carlo optimization applied to a large travelling salesman problem. International Journal of Systems Science DOI: 10.1080/00207729008910387 Volume 21, Issue 3.

[14] Cordeau, Jean-Francois Gendreau, M, and Laporte G (1997) A Tabu Search Heuristic for Periodic and Multi-
Depot Vehicle Routing Problems. Inc. Networks 30: 105 -119 .

[15] Fisher.M. L. and R. Jaikumar.(1981) “A Generalized Assignment Heuristic for Vehicle Routing". Networks, Vol.11, pp.109-124.

[16] Ganesh, K and Narendran, T.T. (2007) 'CLOVES: A cluster-and-search heuristic to solve the vehicle routing problem with delivery and pick-up', European Journal of Operational Research, Volume 178, Issue 3, Pages 699-717

[17] Gilbert and Hofstra,(1992) "A Multiperiod Multiple Traveling Salesman Problem with Heuristic and Application to a Scheduling Problem," Decision Sciences.

[18] Gillett, B., and Miller, L; (1974). "A Heuristic Algorithm for the Vehicle Dispatch Problem," Operations Research, Vol.22, pp.340-350.

[19] Golden, B.L; A.A. Assad, L. Levy, and F.G. Gheysens.(1984) The fleet size and mix vehicle routing problem. Computers \& OR, 11(1):49-66.

[20] Gorenstein S (1970) Printing Press Scheduling for MultiEdition Periodicals http://dx.doi.org/10.1287/mnsc.16.6.B373 Volume 16 Issue 6, pp. B-373-B-383.

[21] Kara, I. and Bektas, T.( 2006) "Integer Linear programming Formulations of Multiple Salesman problems and its variations", European Journal of Operational Research, Vol. 174, No. 3, pp. 1449-1458.

[22] Kolen. A. W. J., Rinnooy Kan. A. H. G and Trienekens. H. W. J. M.(1987) Vehicle Routing with Time Windows http://dx.doi.org/10.1287/opre.35.2.266 OR Volume 35 Issue 2, pp. 266-273.

[23] Laporte, G. and Osman, I.H. (1995) 'Routing problems: a bibliography', Annals of Operational Research, Vol. 61, pp. 227-262.

[24] Lawler, E.L., Lenstra, J.K., Rinnooy Kan, A.H.G., and Shmoys, D.B. (1985) 'The traveling salesman problem: a guided tour of combinatorial optimization', Chichester: Wiley.

[25] Ledesma. J.R and Juan-Jose' Salazar-Gonza'lez (2012) Solving school bus routing using the multiple vehicle traveling purchaser problem: A branch-and-cut approach Computers \& Operations Research 39 (2012) 391-404.

[26] Lenstra, J.K. and Rinnooy Kan, A.H.G. (1975) 'Some simple applications of the traveling salesman problem', Operational Research Quarterly, Vol. 26, pp. 717-733.

[27] Mandell Bellmore, Saman Hong (1974) Transformation of Multisalesman Problem to the Standard Traveling Salesman Problem. Journal of the ACM (JACM), Volume 21 Issue 3, Pages 500-504.

[28] Mitrovic-Minic, S. and Krishnamurti, R ( 2006) "The multiple TSP with time windows: vehicle bounds based on precedence graphs", Operations Research Letters, Vol. 34, No. 1, pp. 111-120 .

[29] Okonjo-Adigwe, C. (1988) 'An effective method of balancing the workload amongst salesmen', Omega, Vol. 16, No. 2, pp. 159-163 
[30] Potvin Jean-Yves and Rousseau Jean-Marc (1995) An Exchange Heuristic for Routing Problems with Time Windows. Journal of the Operational Research Society 46, 1433-1446. doi:10.1057/jors.1995.204.

[31] Parthiban P., P. Venkateswara Reddy, and S. Arunachalam (2011). A review of evolutionary algorithms for multi objective multi product multi modal distribution problem with a possible new approach. Int. J. Logistics economics and globalization vol.3. no.1.

[32] Radharamanan. R., Choi L.I. (1986) A branch and bound algorithm for the travelling salesman and the transportation routing problems. Computers \& Industrial Engineering Volume 11, Issues 1-4, Pages 236-240.

[33] Saleh, H.A. \& Chelouah, R. (2004). The design of the global navigation satellite system surveying networks using genetic algorithms. Engineering Applications of Artificial Intelligence, Vol. 17, pp. 111-22.

[34] Solomon, M. M (1987). Algorithms for the Vehicle Routing and Scheduling Problems with Time Window Constraints. OR, http://dx.doi.org/10.1287/opre.35.2.254 pp. 254-265.
[35] Somhom,S, Modares, A Enkawa T (1999) Competitionbased neural network for the multiple travelling salesmen problem with minmax objective. Computers \& Operations Research 26 395-407.

[36] Svestka, J.A \& Huckfeld, V.E (1973). Computational experience with an $\mathrm{m}$-salesman travelling salesman algorithm . Management Science Vol.19, 790-799.

[37] Tang. L, Liu. j, Rong. A, and Yang. Z (2000) A multiple traveling salesman problem model for hot rolling scheduling in Shanghai Baoshan Iron \& Steel Complex. European Journal of Operational Research 124 , 267-282.

[38] Torki,A, Somhon.S and Enkawa, T (1997) A Competitive Neural Network Algorithm for Solving vehicle Routing Problem. Computers ind. Engng Vol. 33, Nos 3-4, pp. 473-476.

[39] Wang, X and A.C. Regan (2002), Local Truckload Pickup and Delivery with Hard Time Window Constraints, Transportation Research, Part B, Methodological, 36 (2), 97-112. 